Reprod. Nutr. Dévelop., 1987, 27 (1 B), 255-256.

\title{
Effets du niveau d'ingestion et du pourcentage de concentré dans la ration sur la dégradabilité de l'azote in sacco chez le mouton
}

\author{
J. GONZALEZ, Brigitte MICHALET-DOREAU (*), C. PONCET (**)
}

Dpto Alimentacion Animal,

Ciudad Universitaria, 28040 Madrid, Espagne.

(*) Laboratoire des Aliments,

(**) Laboratoire de la Digestion,

I.N.R.A., Theix, 63122 Ceyrat, France.

Summary. The rate of disappearance of nitrogen from 2 feeds - soybean meal and dried alfalfa - was measured with bags incubated in the rumen of sheep receiving, at 2 intake levels, a hay-concentrate diet in different proportions. The theoretical degradability calculated with bag measurements and concentrate retention time in the rumen varied with the proportion of concentrate in the diet and the level of feeding.

La dégradabilité de l'azote alimentaire dans le rumen dépend non seulement des caractéristiques physico-chimiques de l'aliment, mais également de l'intensité et de la durée de l'attaque microbienne. Le présent travail a pour but d'étudier les variations de la cinétique de dégradation in sacco de l'azote de 2 aliments, tourteau de soja et luzerne déshydratée, en fonction du pourcentage de concentré de la ration et des quantités d'aliments ingérées.

Matériel et méthodes. Trois moutons munis d'une canule du rumen et du duodénum ont reçu des rations composées de foin et de concentré dans les proportions suivantes : $90 / 10,70 / 30$ et $40 / 60$. Ces rations ont été distribuées en 8 repas par jour à intervalle de $3 \mathrm{~h}$, à 2 niveaux d'ingestion : au voisinage de l'entretien ( $40 \mathrm{~g} \mathrm{MS} / \mathrm{kg} \mathrm{P}^{0,75}$ ) et à volonté, sauf pour la ration riche en foin (90/10).

Des sachets (pores de $46 \mu \mathrm{m}$ ) contenant $3 \mathrm{~g}$ de tourteau ou de luzerne (broyés à la grille de $0,8 \mathrm{~mm}$ ) ont été placés pendant 2-4-7-17-24 et $48 \mathrm{~h}$ dans le rumen. A chaque durée correspondaient 6 mesures ( 3 animaux $\times 2$ répétitions). Les quantités de matière sèche et d'azote résiduels ont été déterminées sur chaque sachet. La cinétique de dégradation a été décrite avec le modèle mathématique utilisé par Orskov et Mc Donald (1979) $D(t)=a+b\left(1-e^{-c t}\right)$ où $a$ et $b$ représentent respectivement la fraction d'azote alimentaire rapidement disparue par solubilisation et la fraction potentiellement dégradable par les microorganismes du rumen, et où c correspond à la vitesse de dégradation.

Résultats et discussion. La fraction d'azote insoluble et non dégradée au bout de $48 \mathrm{~h}$ par les microorganismes du rumen est indépendante du pourcentage de concentré et du niveau d'ingestion $(23,1$ pour la luzerne et 1,4 pour le tourteau de soja). La répartition entre les fractions immédiatement soluble (a) et potentiellement dégradable (b) est modifiée pour le tourteau de soja (b passe de 85,0 à 77,6 quand le pourcentage de concentré augmente) alors qu'elle reste inchangée pour la luzerne (b est en moyenne égal à 43,5 ) ; mais ces variations ne sont probablement qu'un artefact du modèle mathématique utilisé, à relier aux variations de la vitesse de dégradation (c). Quel que soit l'aliment considéré, cette vitesse augmente de façon importante avec l'augmentation du pourcentage de fourrage et du niveau d'ingestion. Ces modifications traduisent les variations de synthèse 
bactérienne, et donc de capacité protéolytique du rumen; la croissance bactérienne est en effet fonction du taux de renouvellement du rumen (Isaacson et al., 1975) qui dépend lui-même du niveau d'ingestion et du pourcentage de fourrage dans la ration (Harrison et Mc Allan, 1979).

Si on admet pour l'aliment étudié, une vitesse de sortie des particules ( $k$ ) de $0,05 \cdot \mathrm{h}^{-1}$ quelles que soient les conditions d'alimentation du mouton, la dégradabilité théorique $\left(D=a+\frac{b c}{c+k}\right)$ varie dans le même sens que la vitesse (c).

Comme l'avait montré Lindberg (1981), elle diminue de façon importante (- 9,5 points) avec l'augmentation du pourcentage de concentré (de 10 à $60 \%)$; par contre elle varie peu $(+1,1$ point $)$ quand, à même pourcentage de concentré, le niveau d'ingestion augmente.

Si on prend en considération la vitesse réellement mesurée sur l'animal (Poncet et al., 1987), D diminue avec l'augmentation du pourcentage de concentré, mais moins que précédemment, respectivement de $-4,1$ points pour le soja et - 6,7 points pour la luzerne (fig. 1). D diminue également avec l'augmentation du niveau d'ingestion ; l'importance de cette diminution varie avec la nature de la ration. A $30 \%$ de concentré, D diminue d'environ 5 points, soit des résultats voisins de ceux obtenus par Ganev et al. (1979) avec une ration constituée uniquement de fourrage, alors que $D$ n'est pas modifiée avec la ration plus riche en concentré $(60 \%)$.
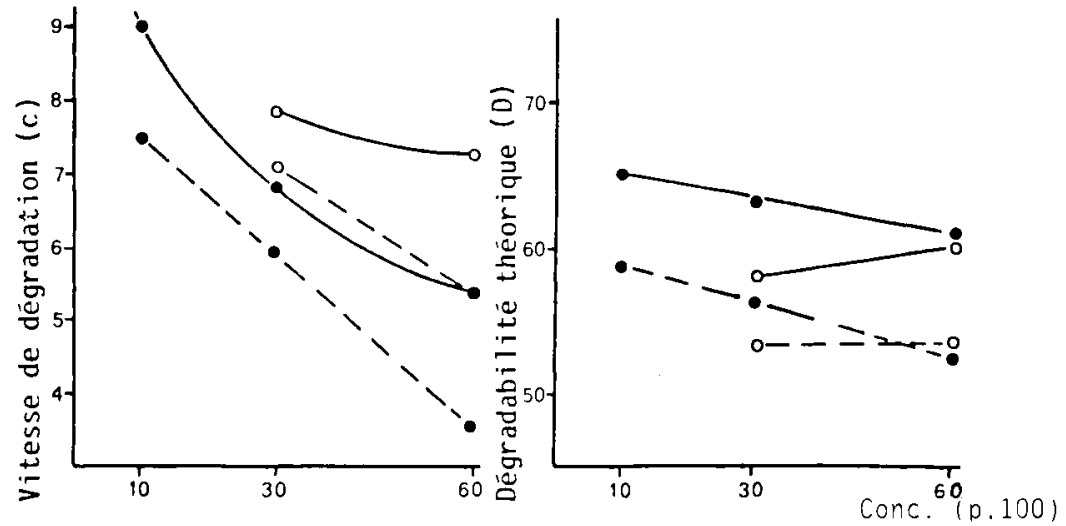

FIG. 1. - Influence du niveau d'ingestion (- limité et o ad libitum) et du pourcentage de concentré sur $C$ et $D$ (calculée à partir de la vitesse de sortie réelle des particules), du tourteau de soja (-) et de la luzerne déshydratée (--).

Comme l'ont mis en évidence de nombreux travaux, les modifications de la composition de la ration de l'animal porteur de sachets entraînent des variations importantes de la dégradabilité de l'azote des aliments, mesurée in sacco, mais ces variations sont tamponnées par la prise en compte du temps de séjour réel de l'aliment dans le rumen.

Ganev G., Orskov E. R., Smart R., 1979. J. agric. Sci. Camb., 93, 651-656.

Harrison D. G., Mc Allan A. B., 1979. Proc. 5th int. Symp. on Ruminal Physiology, 205-225.

Isaacson H. R., Hinds F. C., Bryant M. P., Owens F. N., 1975. J. Dairy Sci., 58, 1645-1659.

Lindberg J. E., 1981. Swedish J. agric. Res., 11, 159-169:

Orskov E. R., Mc Donald 1., 1979. J. agric. Sci. Camb., 92, 499-503.

Poncet C., Gonzalez J., Michalet-Doreau B., 1987. Reprod. Nutr. Dévelop., 27, 257-258. 